\title{
Viral recombination blurs taxonomic lines: examination of single-stranded DNA viruses in a wastewater treatment plant
}

\author{
Victoria M Pearson $^{1}{ }^{\text {， S. Brian Caudle }}{ }^{2}$, Darin R Rokyta ${ }^{\text {Corresp. } 1}$ \\ ${ }^{1}$ Department of Biological Science, Florida State University, Tallahassee, Florida, USA \\ 2 Division of Food Safety, Florida Department of Agriculture and Consumer Services, Tallahassee, Florida, USA \\ Corresponding Author: Darin R Rokyta \\ Email address: drokyta@bio.fsu.edu
}

Understanding the structure and dynamics of microbial communities, especially those of economic concern, is of paramount importance to maintaining healthy and efficient microbial communities at agricultural sites and large industrial cultures, including bioprocessors. Wastewater treatment plants are large bioprocessors which receive water from multiple sources, becoming reservoirs for the collection of many viral families that infect a broad range of hosts. To examine this complex collection of viruses, full-length genomes of circular sSDNA viruses were isolated from a wastewater treatment facility using a combination of sucrose-gradient size selection and rolling-circle amplification and sequenced on an Illumina MiSeq. Single-stranded DNA viruses are among the least understood groups of microbial pathogens due to genomic biases and culturing difficulties, particularly compared to the larger, more often studied dsDNA viruses. However, the group contains several notable well-studied examples, including agricultural pathogens which infect both livestock and crops (Circoviridae and Geminiviridae), and model organisms for genetics and evolution studies (Microviridae). Examination of the collected viral DNA provided evidence for 83 unique genotypic groupings, which were genetically dissimilar to known viral types and exhibited broad diversity within the community. Furthermore, although these genomes express similarities to known viral families, such as Circoviridae, Geminiviridae, and Microviridae, many are so divergent that they may represent new taxonomic groups. This study demonstrated the efficacy of the protocol for separating bacteria and large viruses from the sought after SSDNA viruses and the ability to use this protocol to obtain an in-depth analysis of the diversity within this group. 


\title{
Viral recombination blurs taxonomic lines: examination of single-stranded DNA viruses in a wastewater treatment plant
}

\author{
Victoria M. Pearson ${ }^{1}$, S. Brian Caudle ${ }^{2}$, and Darin R. Rokyta ${ }^{1}$ \\ ${ }^{1}$ Department of Biological Science, Florida State University 319 Stadium Dr. \\ Tallahassee, Florida 32306 \\ ${ }^{2}$ Florida Department of Agriculture and Consumer Services, Division of Food Safety, \\ Bureau of Food Laboratories, 3125 Conner Blvd. BIdg. 9, Tallahassee, Florida 32399
}

\section{ABSTRACT}

\begin{abstract}
Understanding the structure and dynamics of microbial communities, especially those of economic concern, is of paramount importance to maintaining healthy and efficient microbial communities at agricultural sites and large industrial cultures, including bioprocessors. Wastewater treatment plants are large bioprocessors which receive water from multiple sources, becoming reservoirs for the collection of many viral families that infect a broad range of hosts. To examine this complex collection of viruses, full-length genomes of circular ssDNA viruses were isolated from a wastewater treatment facility using a combination of sucrose-gradient size selection and rolling-circle amplification and sequenced on an Illumina MiSeq. Single-stranded DNA viruses are among the least understood groups of microbial pathogens due to genomic biases and culturing difficulties, particularly compared to the larger, more often studied dsDNA viruses. However, the group contains several notable well-studied examples, including agricultural pathogens which infect both livestock and crops (Circoviridae and Geminiviridae), and model organisms for genetics and evolution studies (Microviridae). Examination of the collected viral DNA provided evidence for 83 unique genotypic groupings, which were genetically dissimilar to known viral types and exhibited broad diversity within the community. Furthermore, although these genomes express similarities to known viral families, such as Circoviridae, Geminiviridae, and Microviridae, many are so divergent that they may represent new taxonomic groups. This study demonstrated the efficacy of the protocol for separating bacteria and large viruses from the sought after ssDNA viruses and the ability to use this protocol to obtain an in-depth analysis of the diversity within this group.
\end{abstract}

Keywords: High-throughput Sequencing, Metagenomics, Microviridae, Circoviridae, Geminiviridae, Viral Diversity

\section{INTRODUCTION}

The majority of genomic diversity is contained in viral genomes, yet only a fraction of this diversity has been described (Hatfull, 2008; Thurber, 2009; Clokie et al., 2011). By filling in gaps on these abundant pathogens, we will be better equipped to handle future outbreaks. Advanced knowledge of the natural mutational landscape, along with other viral genes present in the environment for potential recombination, could provide background information should related viral genotypes cause outbreaks (Hambly and Suttle, 2005). Viral genomes have already provided us with many genes that we can use for our benefit, including capsid genes for drug delivery systems and bacteriophage lysis genes as antibiotics (Kovacs et al., 2007; Schmitz et al., 2010). Increasing our knowledge of their diversity is necessary to discover new ways in which we can use their genes and gene products (Hatfull, 2008; Godzik, 2011).

Culture-based studies are important to fully understand viruses that can be grown in vitro. Over 100 years of development has decreased the cost of conducting culture-based studies while vastly improving the culturing methods available (Leland and Ginocchio, 2007). Additionally, approval of these techniques by multiple regulatory boards has resulted in broad implementation. However, we can only culture an extremely limited range of viruses and must complement these approaches with the development and implementation of culture-independent methods (Rosario and Breitbart, 2011). Additionally, standard 
Sanger-sequencing for monitoring new viral pathogens is limited as new viral variants cannot be amplified via PCR until appropriate amplification primers have been created. Therefore, it is necessary to explore viral diversity using new sequencing technologies that do not require the ability to culture the viruses or design specific primers (Thurber, 2009). Culture-independent sequencing methods can improve the monitoring of pathogens in public-health facilities by allowing the sequencing of viral variants that cannot be cultured or amplified via PCR until appropriate primers are available and by decreasing the wait time (Svraka et al., 2010; Barzon et al., 2011; Schmieder and Edwards, 2012).

The ssDNA group of viruses is comprised of seven described viral families: Anelloviridae, Circoviridae, Geminiviridae, Inoviridae, Microviridae, Nanoviridae, and Parvoviridae (King et al., 2012). The ssDNA viruses have mutation rates of $10^{-4}-10^{-7}$ /nucleotide site/generation, similar to RNA viruses, and likewise are small, with genomes ranging from roughly 2-6 kb (Holmes, 2010; Benson et al., 2005). Members of this group include common agricultural pests, such as porcine circoviruses 1 and 2, maize streak virus, and beet curly top virus (Fauquet, 2006). Historically, those involved with agriculture were most interested in increasing our knowledge of this group of viruses, however it is now evident that these viruses are pervasive in many environments and therefore deserve more attention (Rosario et al., 2012b).

We sought to uncover members of the three known ssDNA families with circular genomes, Circoviridae, Geminiviridae, and Microviridae, using a protocol biased towards such genomes. The Circoviridae family have genomes ranging from 1.7-4 kb (Benson et al., 2005; King et al., 2012). All known members of this family are animal viruses, and the majority infect birds. Their genome encodes capsid and replication genes and occasionally 1-2 accessory proteins (Cheung, 2012). These genes may be arranged ambisense, with the genes radiating out from the origin in either direction, or unidirectionally (Benson et al., 2005). Geminiviridae have genomes sizes that range from $2.5-3.1 \mathrm{~kb}$, which is within the range of Circoviridae genomes. All known members are plant viruses with bi-directional genomes and 4-6 genes; some members are monopartite and contain four genes and some are bipartite and have six genes split between two capsids (Bradeen et al., 1997). Recombination has previously been observed between Circoviridae and Geminiviridae (Roux et al., 2013). Both the Circoviridae and Geminiviridae families have capsid and replication initiation genes that are conserved within the viral families and a variable number of accessory genes, which are not conserved. The Microviridae family is comprised of four subfamilies: the Gokushovirinae, the Bullavirinae (formally Microvirinae) (Śliwa-Dominiak et al., 2013; Krupovic et al., 2016), the Pichovirinae (Roux et al., 2012), and the Alpavirinae (Krupovic and Forterre, 2011). Bullavirinae have been thoroughly described and are a model system for genetics and evolution studies (Crill et al., 1999; Wichman et al., 1999; Pearson et al., 2012; Caudle et al., 2014). Members of this family are bacteriophage with genomes sizes from $4.4-6 \mathrm{~kb}$. Gokushovirinae have genome sizes from 4.4-4.9 kb and are comprised of nine genes, whereas Bullavirinae have 11 genes and genomes that range from 5.4-6 kb (Fane et al., 2006). Gokushovirinae produce a major capsid protein, which is similar to the F capsid protein produced by the Bullavirinae. Likewise, the two subfamilies use similar replication initiation proteins, gene A in Bullavirinae. The similarities between these genes among these two subfamilies, along with the conserved nature of these genes in the Circoviridae and Geminiviridae, provide support for using these two genes as the major source of comparison for this study.

Wastewater treatment plants (WWTP) are large bioprocessors that receive influent from households, businesses, and occasionally city street drains, making them reservoirs for plant, animal, and microbial viruses from local (environmental run-off) and distant (plant viruses passed through humans) sources (Cantalupo et al., 2011; Kim et al., 2011; Tamaki et al., 2012). This feature, collecting viruses from multiple sources in the environment, makes WWTPs excellent sites for the study of viral diversity, allowing the investigation of many different viromes with a single collection. However, WWTPs do not just collect viruses, they have the potential to release new viruses into the surrounding area as the effluent from WWTPs have been found to contain large numbers of viruses (Rosario et al., 2009). Additionally, the sludge produced during the treatment of wastewater is often used to fertilize nearby fields or sent to landfills, and if not properly disinfected, any pathogens present will be introduced to the crop being fertilized or ingested by wild animals at landfills (Sano et al., 2003). This study seeks to provide an extensive view of the circular ssDNA viruses present in a single WWTP to identify the viral families present in that environment along with the genetic diversity possible in those families. 


\section{METHODS}

\section{Viral Isolation and DNA extraction}

On February 5, 2012, 2.6 L of sewage were collected from the influent side of the aeration tank at Thomas P. Smith Wastewater Treatment Plant in Tallahassee, FL, USA. Immediately following collection, $60 \mathrm{~mL}$ of chloroform was added to destabilize any plasma membranes present in the sample. The sample was then centrifuged for 15 minutes at $5000 \times \mathrm{g}$. Supernatant was transferred to a clean flask, $72 \mathrm{~g}$ of NaCl was added, and the sample was incubated for 1 hour at $4^{\circ} \mathrm{C}$. To further remove solids, the sample was centrifuged for 10 minutes at $10,000 \times g$. Supernatant was transferred to clean $500 \mathrm{~mL}$ bottles containing $39 \mathrm{~g}$ of PEG8000 and gently rocked to bring PEG into solution. Following an overnight incubation at $4{ }^{\circ} \mathrm{C}$, the sample was centrifuged for 20 minutes at $10,000 \times g$ and the supernatant was discarded. The viral pellet was resuspended using $25 \mathrm{~mL}$ suspension media $\left(0.58 \mathrm{~g} \mathrm{NaCl}, 0.2 \mathrm{~g} \mathrm{MgSO}_{4} * 7 \mathrm{H}_{2} \mathrm{O}, 5 \mathrm{ml} 1 \mathrm{M}\right.$ Tris $\mathrm{pH} 7.5,0.1 \mathrm{~g}$ gelatin, $100 \mathrm{ml} \mathrm{H}_{2} \mathrm{O}$ ). The suspension was centrifuged for 5 minutes at $5000 \times g$ to remove any remaining solids. The supernatant was concentrated using Amicon Ultra- 15 centrifugal filter conicals (100,000 MWCO; EMD Millipore) until the total volume was between 500-600 $\mu \mathrm{L}$. Sample was transferred to a clean tube and $100 \mu \mathrm{L}$ of DNase and $100 \mu \mathrm{L}$ DNase buffer (New England Biolabs) was added to remove any free-floating DNA. Sample was incubated for 30 minutes at $37^{\circ} \mathrm{C}$ and then filter concentrated using Spin-X UF 500 filter concentrators (100,000 MWCO; Corning) until total volume was $500 \mu \mathrm{L}$. The entire sample was loaded onto a 5-30\% sucrose gradient and centrifuged in an ultracentrifuge at 24,000 RPM for 110 minutes at $4{ }^{\circ} \mathrm{C}$.

After centrifugation, $500 \mu \mathrm{L}$ fractions were collected from the bottom of the tube, until the gradient was completely drained. An aliquot of $100 \mu \mathrm{L}$ was reserved for plating and the remaining $400 \mu \mathrm{L}$ had the protein degraded and the DNA extracted using a phenol chloroform DNA extraction method. In brief, the sample was heated to $95^{\circ} \mathrm{C}$ for 15 minutes. After cooling to room temperature $10 \mu \mathrm{L}$ of proteinase $\mathrm{K}$ (Invitrogen) was added to each fraction and incubated at $65^{\circ} \mathrm{C}$ for one hour. Following protein degradation 1/10 volume 1:10 phenol:chloroform isoamyl alcohol (IAA) was added, each fraction was vortexed for 60-90 seconds and centrifuged in a bench top centrifuge for 5 minutes at $2000 \times g$. Supernatant was transferred to fresh tube, equal volume of 1:1 phenol:chloroform IAA was added and the previous vortex and centrifugation step was repeated. Supernatant was transferred to a clean tube and equal volume chloroform IAA was added; vortexing and centrifugation was repeated. A standard ethanol precipitation was performed by transferring the supernatant to a clean tube and adding 1/10 volume sodium acetate and $2 \times$ volume $100 \%$ ethanol. Samples were inverted twice and stored overnight at $-20^{\circ} \mathrm{C}$. DNA precipitation was completed by centrifuging samples for 20 minutes at $15000 \times g$ at $4{ }^{\circ} \mathrm{C}$. Supernatant was removed and $400 \mu \mathrm{L} \mathrm{70 \%} \mathrm{ethanol} \mathrm{was} \mathrm{added} \mathrm{to} \mathrm{the} \mathrm{precipitate} \mathrm{and} \mathrm{a} \mathrm{final} 5$ minute centrifugation at $15,000 \times g$ at $4{ }^{\circ} \mathrm{C}$ was conducted. Supernatants were removed and samples dried in a speedvac. The DNA was rehydrated in $50 \mu \mathrm{L} \mathrm{H}_{2} \mathrm{O}$.

\section{Determination of Target Fractions}

WA13, a Microviridae that is easily cultured and maintained in a laboratory setting (Rokyta et al., 2006), was used in a control gradient to identify which fractions contained the target ssDNA genomes. The control gradient was performed using the protocol described above for the sample gradient. Each fraction of both gradients was spread on nutrient rich agar plates using Escherichia coli $\mathrm{C}$ as a host to determine which fractions had the highest number of viable small bacteriophage. Every fraction from the sample gradient also had the DNA quantified on both a Nanodrop spectrophotometer and a QuBit (Life Technologies). The combination of these data was used to determine the six fractions to use for sequencing by correlating the WA13 containing fractions from the control gradient to the comparable DNA peak in the sample gradient.

\section{Amplification, Library Preparation, and Sequencing}

Rolling circle amplification of individual fractions was performed with a Genomiphi V2 kit (GE Healthcare) according to manufacturer's protocol. An ethanol precipitation was conducted on the amplified fractions as described above. Amplified fractions were quantified using a Qubit and aliquots of each amplified fraction were made containing at least $1 \mu \mathrm{g}$ DNA; $\mathrm{H}_{2} \mathrm{O}$ was then added to a final volume of $200 \mu \mathrm{L}$. The fractions were sonicated in a Bioruptor (Diagenode) for 9 cycles of 30 seconds on high, 30 seconds off in order to fragment the DNA. Fractions were dehydrated and resuspended in $50 \mu \mathrm{L}$ $\mathrm{H}_{2} \mathrm{O}$. Library preparation for a 300 cycle sequencing run was done using an Illumina TruSeq DNA Kit 
according to the manufacturers specifications. Individual fractions were multiplexed for identification after sequencing. Prepared samples were diluted to $10 \mathrm{nM}$ and pooled together, final product was diluted to $2 \mathrm{nM}$. Sequencing was conducted on an Illumina MiSeq by loading 9 picomoles into the Illumina sequencing cartridge.

\section{Data Analysis}

Sequencing reads from the individual fractions were de novo assembled using SeqMan NGen (version 11) by DNAStar. All contigs greater than $2000 \mathrm{nt}$ long with at least $3 \mathrm{X}$ coverage were analyzed for circularity by looking for repeats at the beginning and end of the sequence. Circular contigs were considered full-genomes and were trimmed to remove duplicate ends. Full genome contigs were annotated using BLASTx for gene matches. Long open reading frames that did not get a hit on BLASTx were annotated as hypothetical genes. Pairwise comparisons were completed to remove duplicate genomes. For the determination of how many sequencing reads belonged to each viral group and to ensure accurate representation of single nucleotide polymorphisms (SNP) frequency, a second assembly was conducted using the annotated contigs as templates. The default parameters in SeqMan NGen for a metagenomic templated assembly, except no limitations were placed on deep regions was used. The SNPs were identified using SeqMan and considered valid when present in at least $20 \%$ of the mapped reads. The nonsynonymous (NS) SNPs were identified by pairwise gene comparisons between the consensus amino acid (AA) sequence and the resulting AA sequence with the SNP changes. Pairwise comparisons of the AA sequence of each gene to both their individual top hit from the BLASTx searches, and to all of the other members of their grouping in the community were performed to determine the percent identity in MegAlign by DNAStar. Cluster analysis was performed in R using the mclust package (R, 2010). Mclust was allowed to perform model selection to determine the best fit model and identify putative clusters (Fraley and Raftery, 2002). A genetic pairwise identity matrix was constructed for all individuals from each group for the capsid and replication genes. Raw sequencing reads have been uploaded to the sequence read archive (SRR3580070). All of the genotypes have been uploaded to NCBI (Circo/Geminiviridae: KX259394-KX259454; Microviridae: KX259455-KX259476).

\section{RESULTS}

\section{Community Composition}

All complete genomes with sequence similarities to the Microviridae family were most similar to the Gokushovirinae subfamily. The remaining whole genomes shared sequence similarities with either the Circoviridae or Geminiviridae families, or shared sequence similarities with both families, in the form of having one gene most closely related to the Circoviridae family and another gene most closely related to the Geminiviridae family. Due to the high level of recombination or intermediate forms in the Circoviridae and Geminiviridae families, all of the genomes that appeared similar to the Circoviridae or Geminiviridae families were combined together as one group (Figure 1). Of the 7,723,150 total sequencing reads, 6,224,682 assembled into whole and partial genomes (whole: 5,118,276, partial: $1,106,406)$, leaving $1,498,468$ that did not assemble, during a final templated assembly using the described genotypes as the references. The majority of whole genome $(4,860,112$ reads) and partial genome length contigs were members of the Circo/Geminiviridae group, accounting for 5,326,390 reads of sequencing assembled into 61 genotypes (Figure 2). The remaining 22 whole genomes (258,164 reads) along with some of the partial genome length contigs were Microviridae, accounting for 319,493 of the sequences. Relatively few sequences were determined to be bacterial in origin (164,845 reads), and they were all short fragments of genomes. The remaining 413,954 sequences were either unknown, meaning that BLASTx searches did not provide any matches, or unclassified, indicating that the hits from BLASTx searches were to uploaded sequences of unknown origins and could be prokaryotic, eukaryotic, or viral. Of the $61 \mathrm{Circo} / \mathrm{Geminiviridae}$ genotypes, 32 contained two open reading frames (ORFs), 22 contained three ORFs, and seven had four ORFs. The capsid gene was annotated as hypothetical for 30 of the Circo/Geminiviridae genotypes as only the replication gene had a match on GenBank. Eighteen of the capsid genes were of Circoviridae ancestry, four were of Geminiviridae ancestry, and eight had top matches that were unclassified. The replication gene was annotated as hypothetical for one of the Circo/Geminiviridae genotypes as only the capsid gene had a match on GenBank. Thirty two of the replication genes were of Circoviridae ancestry, 12 were of Geminiviridae ancestry, and 16 had top matches that were unclassified. All of the Microviridae genotypes were most closely related to the 

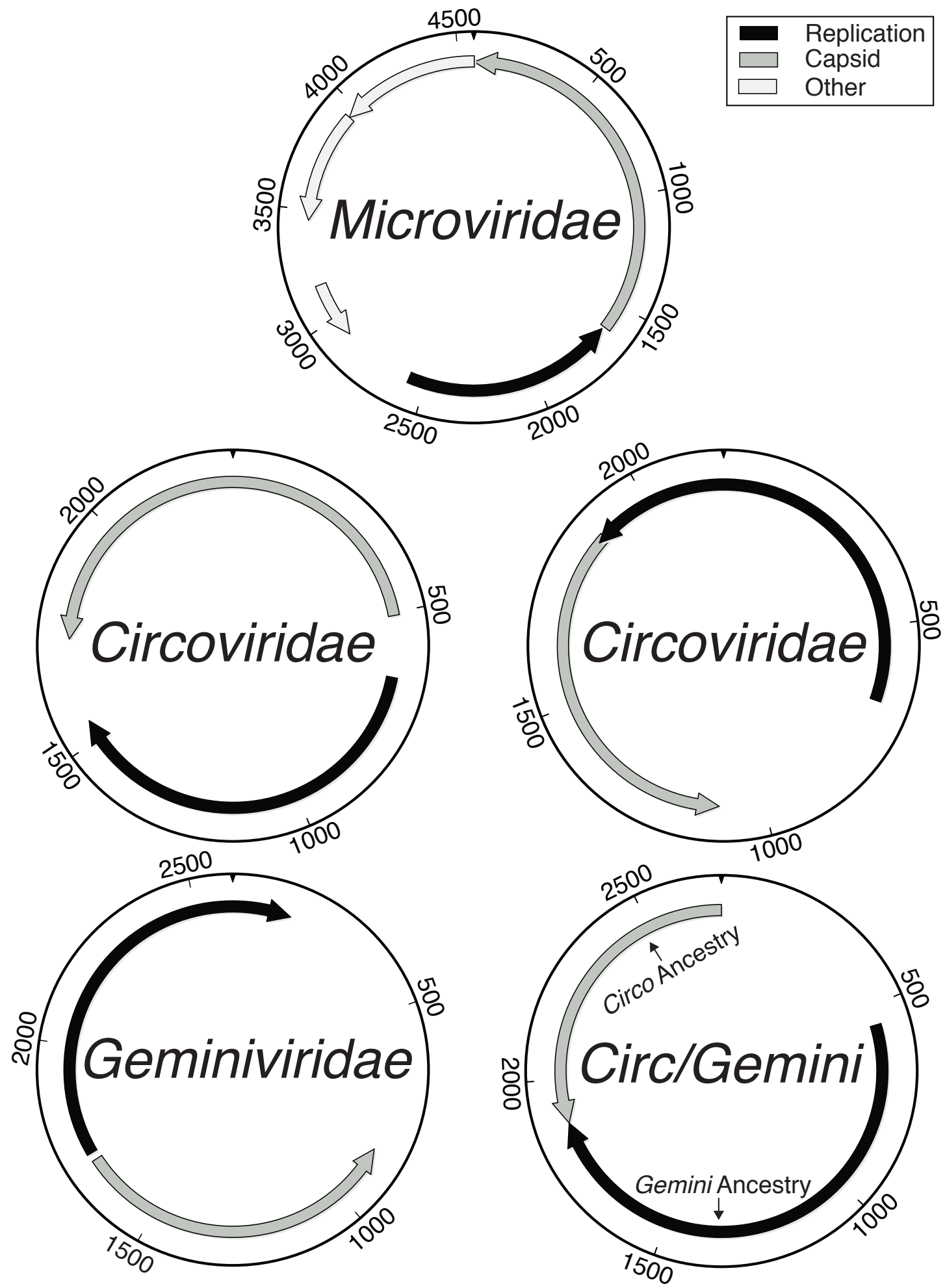

Figure 1. Genome maps demonstrating the ambiguity of the Circoviridae/Geminiviridae genomes. Maps labeled as Circoviridae were examples of genotypes where both main genes had Circoviridae origins and were either uni- or bi-directional. All Geminiviridae genomes were bi-directional. The Circ/Gemini map displays an example of recombination, where each main gene has a different ancestral origin and the genomic organization is bi-directional. Although Microviridae have 9-11 genes, the novelty of the genotypes found limited the amount of genes that could be accurately annotated. 


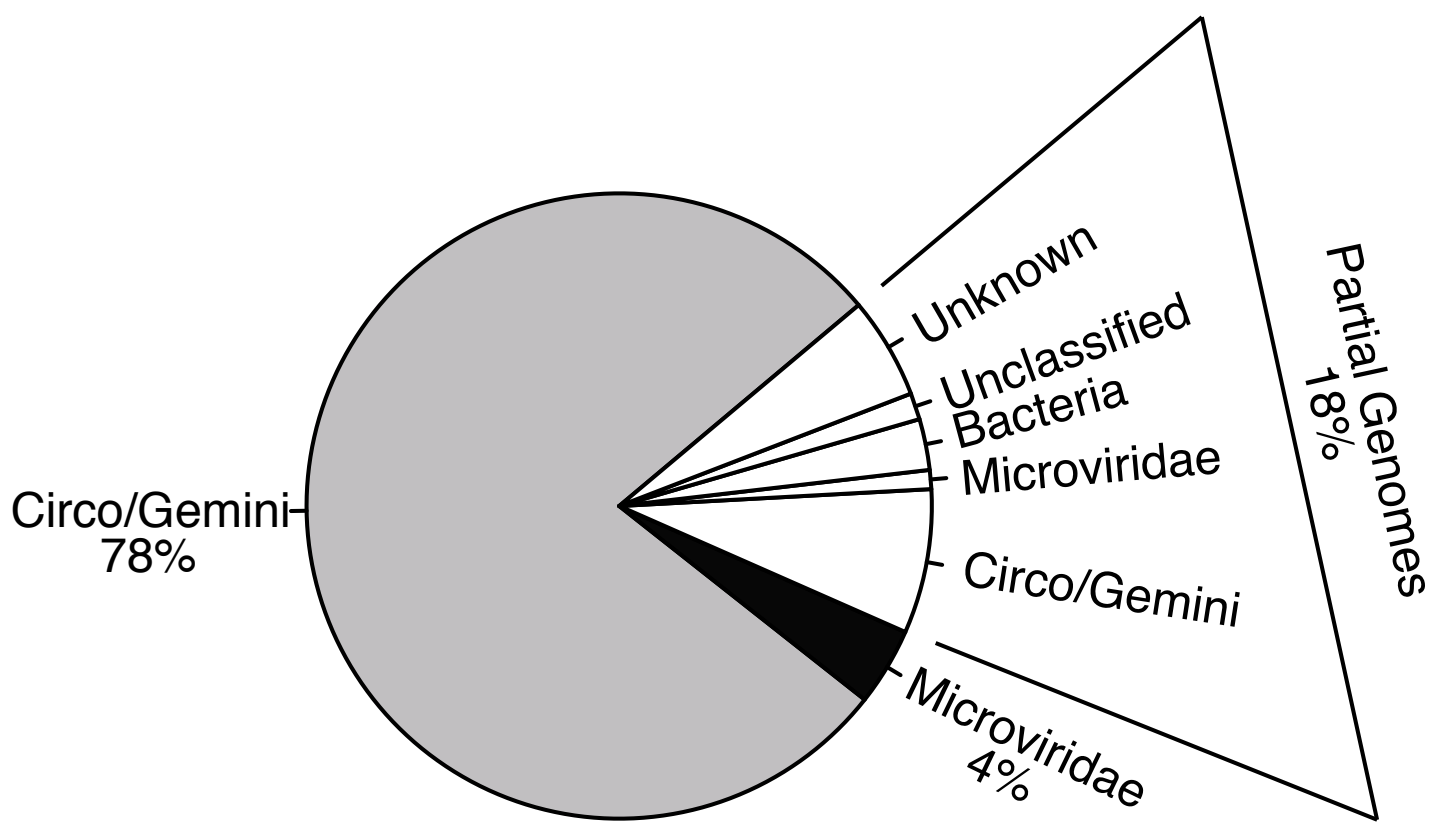

Figure 2. Distribution of sequencing effort demonstrates the efficacy of the protocol for isolating the ssDNA viruses. Few sequences were recovered from bacterial contaminants and all were partial genomes (white). The majority of the sequences assembled into whole genomes from the combined Circo/Geminiviridae group (grey) and the Microviridae family (black) during a reference based assembly using the found genotypes as templates.

Gokushovirinae subfamily for both the capsid and replication genes and did not contain any unexpected genes.

Pairwise comparisons were conducted for both the replication initiation (REP) and capsid gene AA sequences for each genotype against all of the other genotypes in their groupings to determine the percent identity between all of the genotypes in the sample. The majority of comparisons from the Circo/Geminiviridae group demonstrated that the pairs were less than 50\% similar to each other, with very few isolated comparisons demonstrating a higher percent of identity (Figure 3). The Microviridae group once again demonstrated a higher level of identity between members of their own community, however the majority of sequences were still below $60 \%$ identity. Cluster analysis identified nine clusters within the Circo/Geminiviridae (diagonal, equal shape model; BIC: -28847.64) group and seven clusters within the Microviridae (diagonal, varying volume and shape model; BIC: -3358.822) group (Figure 3).

\section{Intercommunity Diversity}

Pairwise comparisons between the amino acid sequences for the REP and capsid genes from the recovered genomes and their top matches on BLASTx revealed that all of the recovered genes were $<70 \%$ similar to their top hit (Figure 4). Although all of the recovered genes only shared minimal AA identity with previously described viral genotypes, there were several that either had a common top match from BLASTx, or that shared top matches from the same study. Several members of the Circo/Geminiviridae group shared some AA sequence identity to genotypes discovered during other metagenomic studies in rodent stools (Phan et al., 2011), dragonflies (Rosario et al., 2012a) and from seawater collected nearby in Tampa Bay, Florida (Mcdaniel et al., 2013). Interestingly, several members of the Microviridae group also shared top matches with genotypes discovered in dragonflies (Rosario et al., 2012a). Multiple Microviridae group members also shared some AA sequence identity with genotypes discovered in ocean environments (Labonté and Suttle, 2013a). In the Circo/Geminiviridae group, it was common for one gene (in all but one case the REP gene) to have a match, but for the capsid gene to have no match on GenBank, resulting in many genomes having their capsid gene aligned at the $0 \%$ identity line. In the Microviridae group both genes had a match on GenBank and all genes demonstrated at least 30\% AA 

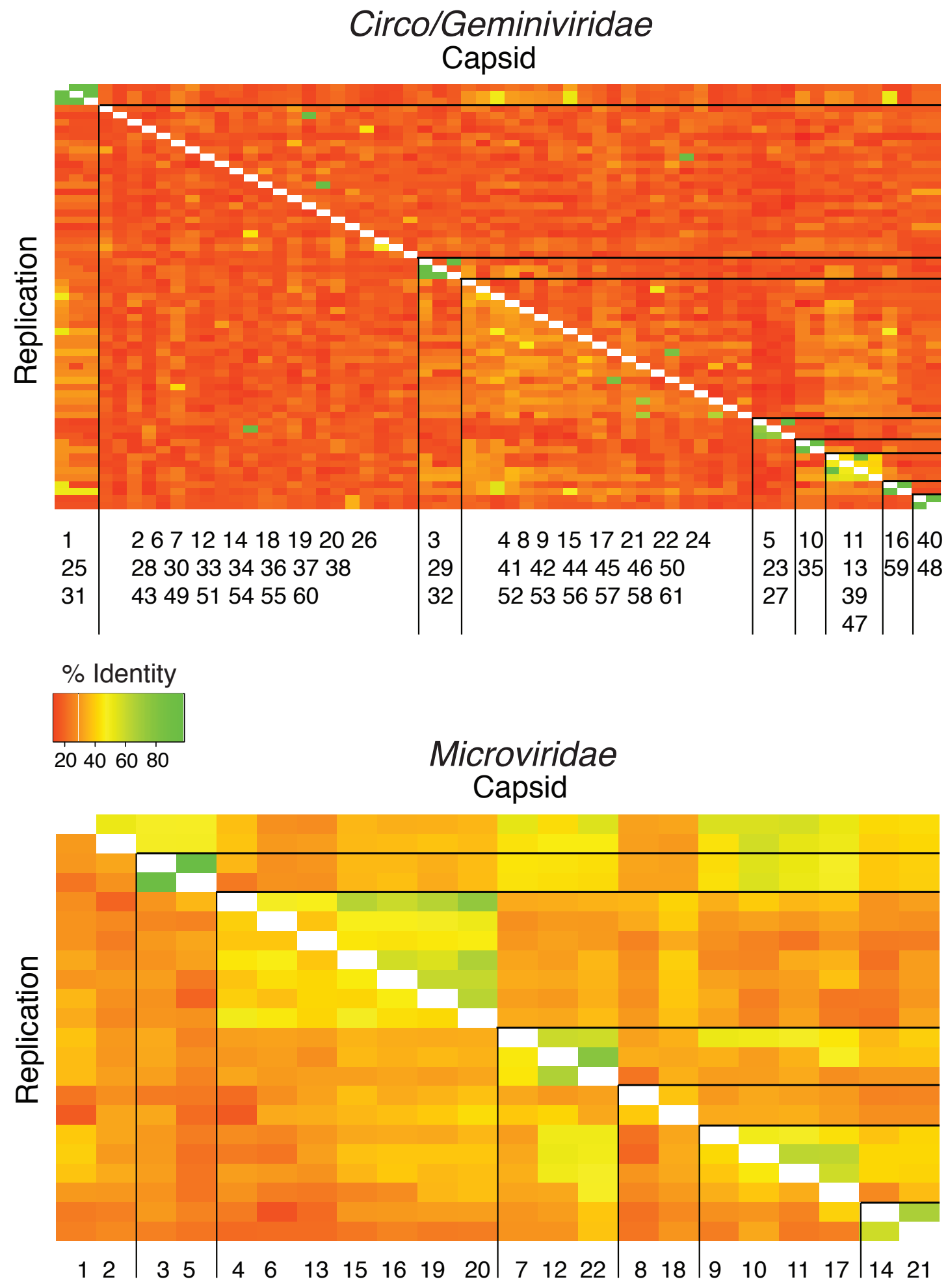

Figure 3. Heat maps visualize pairwise comparisons for the capsid and replication genes AA sequences between each genotype in the population. Very few genotypes within the WWTP are similar (more green), exhibiting a high level of dissimilarity (more orange). The genotypes are grouped based on cluster analysis into groupings of AA sequence identity using both capsid and REP genes. These groups may represent subfamilies and genera. The genotypes are listed along the $\mathrm{X}$-axis within the cluster group in which they belong. The genotype numbers correspond to their official names on GenBank. 

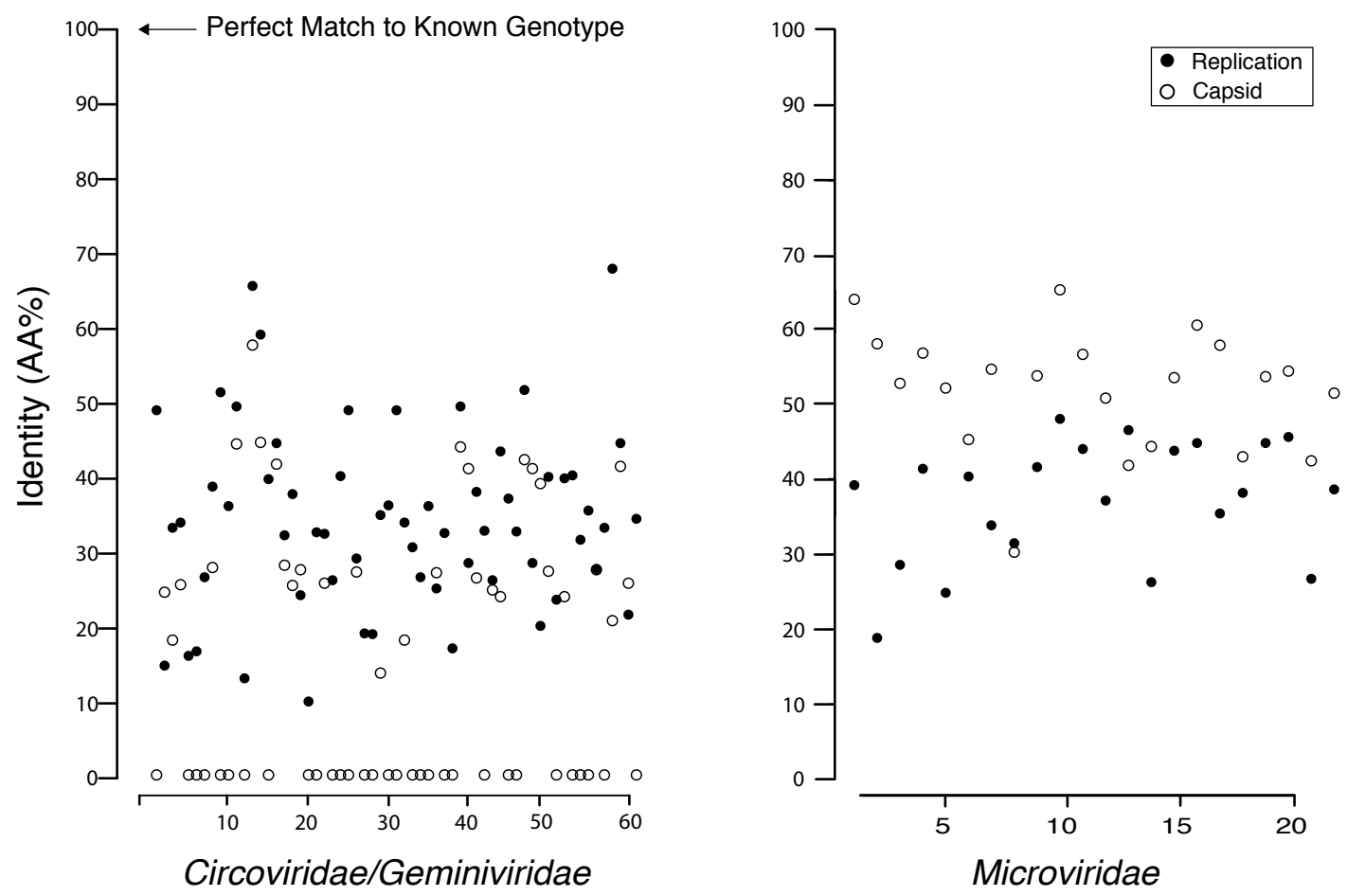

Figure 4. Percent identity between the capsid and replication genes for the discovered genotypes and their closest match from GenBank, which shows that all of the found genes are extremely dissimilar from their closest known relative. Many of the Circo/Geminiviridae genotypes had one gene that did not have a match on GenBank and is therefore completely dissimilar to known viral genes.

sequence identity.

\section{Genetic Polymorphisms}

The total number of SNPs within a genome were used to determine if the contig was an assembly of one or multiple potential genotypes. For both the Circo/Geminiviridae and Microviridae groups, there were a few contigs that did not have any SNPs above the reported cut-off and may be comprised of a single genotype. The remaining genomes contain multiple SNPs and can therefore be considered assemblages of closely related genotypes (Figure 5). In the Circo/Geminiviridae group, half of the genotypes had between $0.0075-0.0113 \mathrm{SNP} /$ nucleotide, whereas in the Microviridae group most of the genotypes had between 0-0.0038 SNP/nucleotide. For both the capsid and REP genes, the NS SNP frequency was determined (Figure 6). Comparisons of the number of NS SNPs between the capsid and REP genes within both the Circo/Geminiviridae and Microviridae showed no significant differences (Welch's t-test, $p=0.2$ and 0.5 respectively).

\section{DISCUSSION}

We examined the diversity of ssDNA viruses in a wastewater treatment plant, by means of whole-genome sequencing. Previous studies have examined the phage diversity in WWTP using culture-based methods, which biased the results to only finding viruses that can be grown in vitro (Rokyta et al., 2006). Attempts to remove this bias have included shotgun sequencing to get a rough description of the diversity, however, these studies mainly focused on the effluent leaving the treatment facilities or were biased towards dsDNA viruses (Rosario et al., 2009; Parsley et al., 2010; Tamaki et al., 2012). Other ssDNA virus diversity studies have focused on rice paddy soil and dragonflies using cloning techniques and Sanger sequencing (Kim et al., 2008; Rosario et al., 2012a). Additional environmental studies reduced circular genome bias while investigating viromes in ocean water and human feces using 454 (Kim et al., 2011; Labonté 


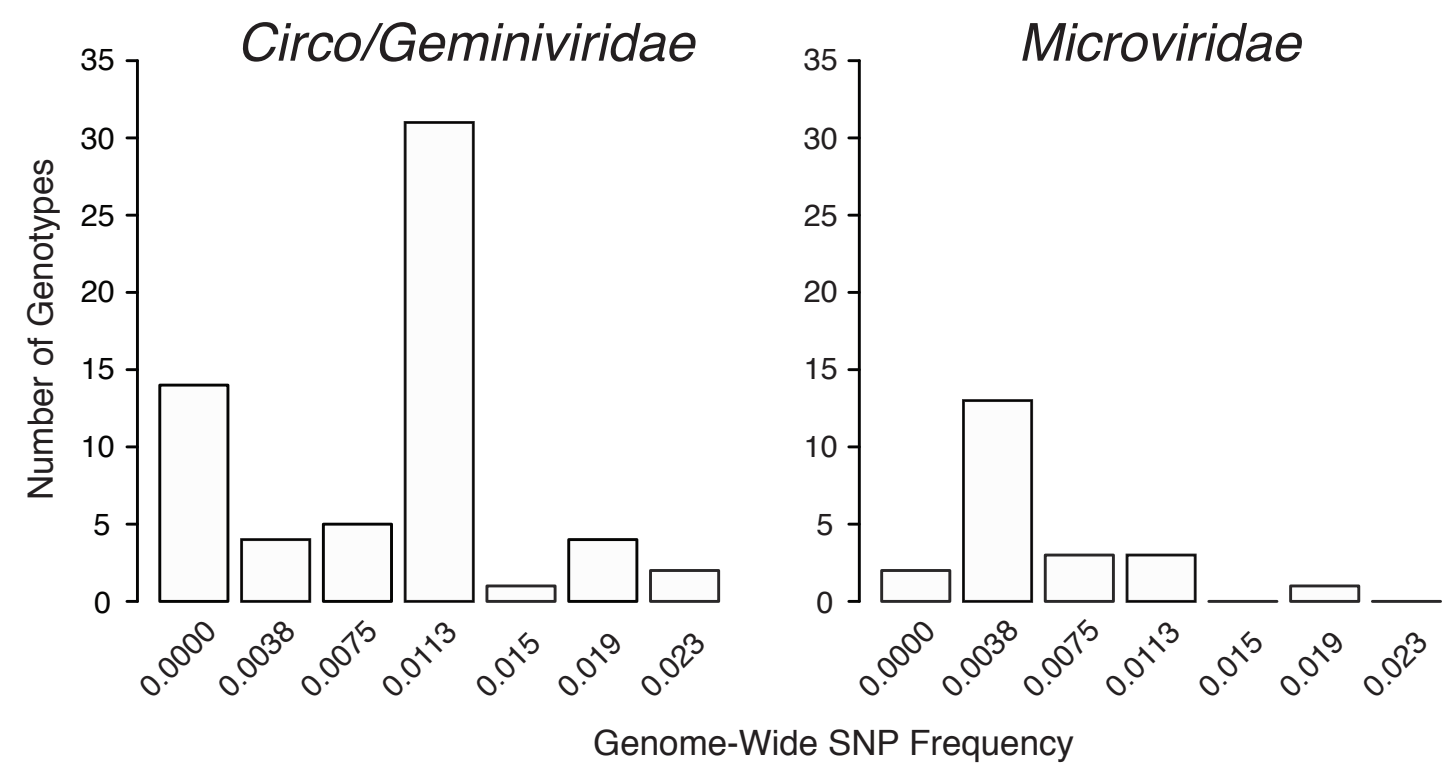

Figure 5. Distribution of SNP frequencies within identified genotypes. Total number of genotypes containing SNPs at binned frequencies illustrates that many are actually genomic assemblages.
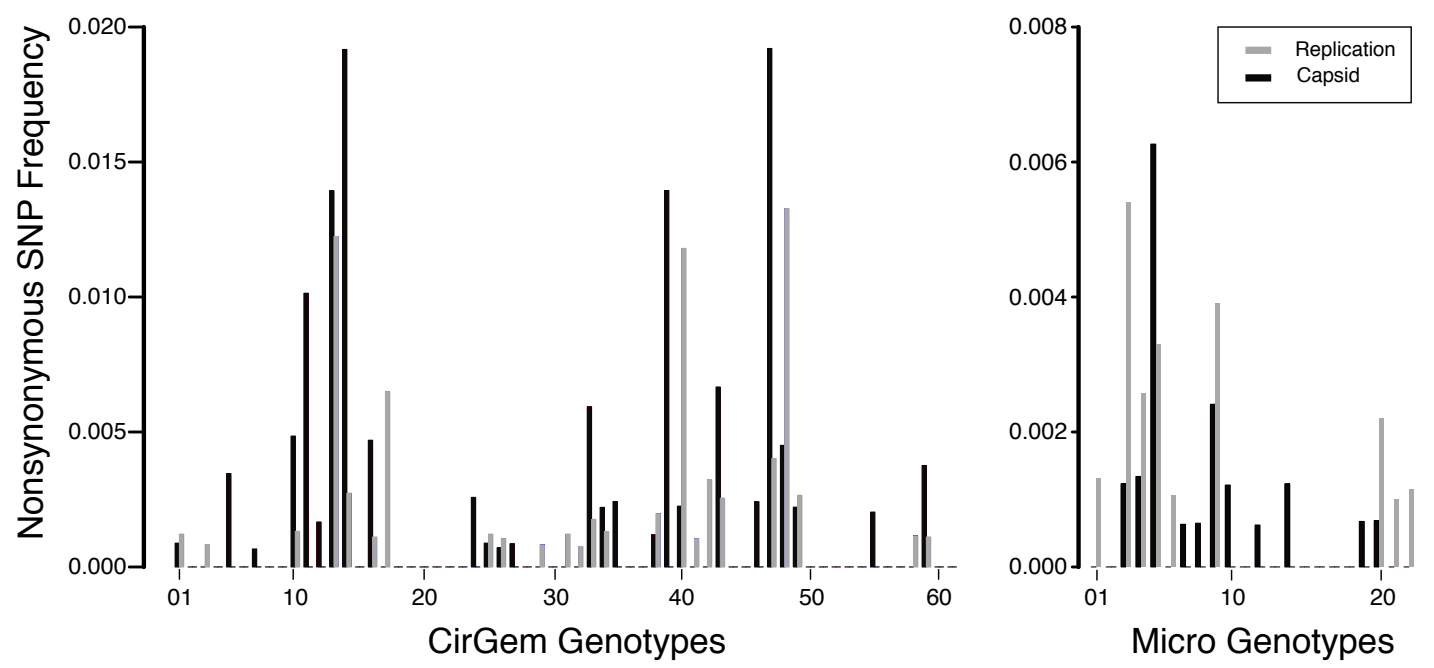

Figure 6. Nonsynonymous SNP frequency for the capsid and replication genes, demonstrating that the capsid and REP genes experience varying amounts of change in different genetic backgrounds. 
and Suttle, 2013b). In this study, size-selection methods and amplification biases (Kim and Bae, 2011) minimized bacterial contamination and completely remove all large dsDNA and RNA viruses from the sample, focusing the sequencing effort on ssDNA viruses. This method allowed for the ascertainment of information on all of the circular ssDNA viral families present in the system by removing the limitation of only recovering phage genomes.

Wastewater treatment plants rely on microorganisms to break down the organic matter present in the wastewater, and, as such, the viruses that infect these microbes are often abundant throughout the system (Shapiro and Kushmaro, 2011). Therefore, this study was initiated with the goal of investigating mostly Microviridae bacteriophage and potentially a few non-phage ssDNA viruses. By removing any culturing biases, we discovered a higher number of plant and animal virus genomes than bacteriophage, even though these are likely transient in the system. This conundrum of detecting more eukaryotic viruses than phage could represent bias of rolling circle amplification, because the Circoviridae and Geminiviridae-like viruses are much smaller than the Microviridae and should therefore amplify more quickly. Alternatively, it could be that the majority of ssDNA viruses are eukaryotic viruses, and this study uncovered an accurate portrayal of the percentage of ssDNA eukaryotic viruses versus prokaryotic viruses in the WWTP. Previous studies examining prokaryotic ssDNA viruses in WWTP have found that they are present in much lower numbers than the larger dsDNA viruses (Rokyta et al., 2006). Therefore, the genetic structure and limited gene composition of ssDNA viruses may make them better suited for infecting eukaryotic rather than prokaryotic hosts. The approach presented here has allowed a much broader taxonomic sampling than previously expected by facilitating characterization of the full genomic community of circular ssDNA viruses in the WWTP.

We found viral genomes with similarities to the known viral families Circoviridae, Geminiviridae, and Microviridae. However, for the Circo/Geminiviridae like genotypes, the AA sequence identity of both the capsid and REP gene, is $<70 \%$ for all genotypes. Further, the AA sequence identity of all the capsid genes is $<60 \%$, and for many is $<20 \%$, indicating that they may all belong to new viral genera and subfamilies, demonstrated by the groupings assembled by cluster analysis (Rosario et al., 2012b). As the current known viral families on GenBank are primarily based upon viruses that have been discovered via standard culturing techniques, it is not surprising that multiple recent metagenomic studies have claimed the newly discovered genomes from their studies belong to new viral taxonomic groups of various levels ( $\mathrm{Ng}$ et al., 2009; Rosario and Breitbart, 2011; Phan et al., 2011). More remarkable is that each metagenomic study has found new viral genera and families not present in the others, demonstrating the limited nature of the current understanding of viral taxonomic diversity (van den Brand et al., 2012). Pairwise comparisons between all of the viruses identified determined that the majority are dissimilar from one another. Further, the SNP analysis showed that there are an unknown number of virus genomes assembled into the majority of genotypes in this study. Therefore, what this study refers to as genotypes may be groups of very similar viruses within these new taxonomic groups and more accurately be described as species rather than genotypes.

Several genotypes discovered were found to have one gene that was most closely related to one viral family (Circoviridae) while another gene was similar to a different viral family (Geminiviridae). We propose these to be recombination events, not relics of improper assembly, as inspection of the assembly files showed level coverage across these genomes. Recombination events between both closely related viruses (Rokyta et al., 2006; Lefeuvre et al., 2009; Roux et al., 2013) and distantly related viral families has been reported with ssDNA viruses (Diemer and Stedman, 2012; Krupovic et al., 2015). As these genotypes are already dissimilar from the known viral genotypes within these families, it is unclear when these recombination events occurred. The recombined genotypes may represent continual recombination that is occurring between two closely related, but previously unknown viral groups. If this is the case, these new viruses potentially infect the same range of hosts, unlike the two viral families to which they are similar, which infect animals and plants, and may not have the opportunity for continual recombination. Conversely, this could be indicative of an ancient recombination event that occurred once or relatively few times, and diversification of the resulting recombinants led to the formation of these new viral groups that we are uncovering now. These recombinant genotypes may belong within either the Circoviridae or Geminiviridae families, or may represent a new viral family.

Wastewater treatment plants require microbes to properly digest and treat the influent, therefore viruses that infect these microbes are abundant in the system (Sano et al., 2003; Parsley et al., 2010; Cantalupo et al., 2011). The viral pathogens present do not aid in the digestion of the organic matter and may be 
hindering the digestion by interfering with the desired microbial populations (Shapiro et al., 2009). Only a small proportion of the recovered genotypes were closely related to known bacteriophage, however the majority of the recovered viral genotypes were too distantly related to any known genotypes as to be sure of their host range. The Circoviridae and Geminiviridae families are eukaryotic viruses that infect a broad spectrum of animals and plants respectively (Bradeen et al., 1997; Cheung, 2012; King et al., 2012). Therefore it is likely that our Circo/Geminiviridae like viruses are infecting eukaryotic hosts, but the host may not be the same as its closest match on GenBank. It is possible that the new viral groups are not merely washing into and out of the system, but rather are infecting unknown hosts that are permanently present within the system. Methods to elucidate the host of these viruses without culturing are needed to determine if these are transient viruses that infect plants and animals such as their distant relatives, or whether these new viruses are in fact residents of the WWTP. Alternatively, monitoring these viral populations could provide further support as to their stability in the system. Genotypes recovered across multiple time points could indicate permanent association with the WWTP; conversely limited sampling recovery provides support for transiency and minimal importance to the overall WWTP operation.

\section{ACKNOWLEDGMENTS}

We thank Margaret Seavy and Dr. Steven Miller for technical assistance throughout the processing of the sample, Dr. Lindsey McGee for providing guidance with SNP analysis, and Sarah Lueking and Kate Hill for providing writing guidance. We thank the employees of the Thomas P. Smith Water Reclamation Facility for assisting with sample collection.

\section{REFERENCES}

Barzon, L., Lavezzo, E., Militello, V., Toppo, S., and Palù, G. (2011). Applications of next-generation sequencing technologies to diagnostic virology. Int J Mol Sci, 12(11):7861-84.

Benson, D., Karsch-Mizrachi, I., Lipman, D., Ostell, J., and Wheeler, D. (2005). Genbank. Nucleic Acids Research, 33(suppl 1):D34-D38.

Bradeen, J., Timmermans, M., and Messing, J. (1997). Dynamic genome organization and gene evolution by positive selection in geminivirus (geminiviridae). Molecular biology and evolution, 14(11):11141124.

Cantalupo, P. G., Calgua, B., Zhao, G., Hundesa, A., Wiler, A. D., Katz, J. P., Grabe, M., Hendrix, R. W., Girones, R., Wang, D., and Pipas, J. M. (2011). Raw sewage harbors diverse viral populations. mBio, 2(5).

Caudle, S. B., Miller, C. R., and Rokyta, D. R. (2014). Environment determines epistatic patterns for a ssdna virus. Genetics, 196(1):267-279.

Cheung, A. (2012). Porcine circovirus: transcription and dna replication. Virus Research, 164(1):46-53.

Clokie, M., Millard, A., Letarov, A., and Heaphy, S. (2011). Phages in nature. Bacteriophage, 1(1):31-45.

Crill, W. D., Wichman, H. A., and Bull, J. J. (1999). Evolutionary reversals during viral adaptation to alternating hosts. Genetics, 154:27-37.

Diemer, G. S. and Stedman, K. M. (2012). A novel virus genome discovered in an extreme environment suggests recombination between unrelated groups of rna and dna viruses. Biology Direct, 7(13):1-14.

Fane, B., Brentlinger, K., Burch, A., Chen, M., Hafenstein, S., Moore, E., and Novak, C. (2006). Phix174 et al., the microviridae. The Bacteriophages.

Fauquet, C. (2006). The diversity of single stranded dna viruses. Biodiversity, 7(1):38-44.

Fraley, C. and Raftery, A. (2002). Mclust: Software for model-based clustering, density estimation and discriminant analysis. DTIC Document.

Godzik, A. (2011). Metagenomics and the protein universe. Current opinion in structural biology, 21.

Hambly, E. and Suttle, C. (2005). The viriosphere, diversity, and genetic exchange within phage communities. Current opinion in microbiology, 8(4):444-450.

Hatfull, G. F. (2008). Bacteriophage genomics. Current Opinion in Microbiology, 11(5):447-453.

Holmes, E. C. (2010). The comparative genomics of viral emergence. Proceedings of the National Academy of Sciences, 107(suppl 1):1742.

Kim, K.-H. and Bae, J.-W. (2011). Amplification methods bias metagenomic libraries of uncultured singlestranded and double-stranded dna viruses. Applied and Environmental Microbiology, 77(21):76637668 . 
Kim, K.-H., Chang, H.-W., Nam, Y.-D., Roh, S. W., Kim, M.-S., Sung, Y., Jeon, C. O., Oh, H.-M., and Bae, J.-W. (2008). Amplification of uncultured single-stranded dna viruses from rice paddy soil. Applied and environmental microbiology, 74(19):5975-5985.

Kim, M.-S., Park, E.-J., Roh, S. W., and Bae, J.-W. (2011). Diversity and abundance of single-stranded dna viruses in human feces. Applied and environmental microbiology, 77(22):8062-8070.

King, A. M. Q., Adams, M. J., and Lefkowitz, E. J. (2012). Virus taxonomy: classification and nomenclature of viruses: Ninth report of the international committee on taxonomy of viruses. Elsevier.

Kovacs, E. W., Hoker, J. M., Romanini, D. W., Holder, P. G., Berry, K. E., and Francis, M. B. (2007). Dual-surface-modified bacteriophage $\mathrm{ms} 2$ as an ideal scaffold for a viral capsid-based drug delivery system. Bioconjugate Chemistry, 18(4).

Krupovic, M., Dutilh, B. E., Adriaenssens, E. M., Wittmann, J., Vogensen, F. K., Sullivan, M. B., Rumnieks, J., Prangishvili, D., Lavigne, R., Kropinski, A. M., Klumpp, J., Gillis, A., Enault, F., Edwards, R. A., Duffy, S., Clokie, M. R. C., Barylski, J., Ackermann, H.-W., and Kuhn, J. H. (2016). Taxonomy of prokaryotic viruses: update from the ictv bacterial and archaeal viruses subcommittee. Archives of virology, 161(4):1095-1099.

Krupovic, M. and Forterre, P. (2011). Microviridae goes temperate: Microvirus-related proviruses reside in the genomes of bacteroidetes. PloS one, 6(5):e19893.

Krupovic, M., Zhi, N., Li, J., Hu, G., Koonin, E. V., Wong, S., Shevchenko, S., Zhao, K., and Young, N. S. (2015). Multiple layers of chimerism in a single-stranded dna virus discovered by deep sequencing. Genome Biology and Evolution, 7(4):993-1001.

Labonté, J. and Suttle, C. (2013a). Metagenomic and whole-genome analysis reveals new lineages of gokushoviruses and biogeographic separation in the sea. Front. Microbiol., 4.

Labonté, J. M. and Suttle, C. A. (2013b). Previously unknown and highly divergent ssdna viruses populate the oceans. The ISME journal, 7(11):2169-2177.

Lefeuvre, P., Lett, J., Varsani, A., and Martin, D. (2009). Widely conserved recombination patterns among single-stranded dna viruses. Journal of Virology, 83(6):2697.

Leland, D. S. and Ginocchio, C. C. (2007). Role of cell culture for virus detection in the age of technology. Clinical Microbiology Reviews, 20(1):49-78.

Mcdaniel, L. D., Rosario, K., Breitbart, M., and Paul, J. H. (2013). Comparative metagenomics: natural populations of induced prophages demonstrate highly unique, lower diversity viral sequences. Environmental Microbiology, 16(2):570-585.

Ng, T., Manire, C., Borrowman, K., Langer, T., Ehrhart, L., and Breitbart, M. (2009). Discovery of a novel single-stranded dna virus from a sea turtle fibropapilloma by using viral metagenomics. Journal of Virology.

Parsley, L. C., Consuegra, E. J., Thomas, S. J., Bhavsar, J., Land, A. M., Bhuiyan, N., Mazher, M. A., Water, R. J., Wommack, K. E., and Harper, W. F. (2010). A census of the viral metagenome within an activated sludge microbial assemblage. Applied and environmental microbiology, 76(8).

Pearson, V., Miller, C., and Rokyta, D. (2012). The consistency of beneficial fitness effects of mutations across diverse genetic backgrounds. PloS one, 7(8):e43864.

Phan, T. G., Kapusinszky, B., Wang, C., Rose, R. K., Lipton, H. L., and Delwart, E. L. (2011). The fecal viral flora of wild rodents. PLoS Pathog, 7(9):e1002218.

R, D. C. T. (2010). R: a language and environment for statistical computing. Vienna, Austria: $R$ Foundation for Statistical Computing.

Rokyta, D. R., Burch, C. L., Caudle, S. B., and Wichman, H. A. (2006). Horizontal gene transfer and the evolution of microvirid coliphage genomes. Journal of bacteriology, 188(3):1134-1142.

Rosario, K. and Breitbart, M. (2011). Exploring the viral world through metagenomics. Current Opinion in Virology, 1(4):289-297.

Rosario, K., Dayaram, A., Marinov, M., Ware, J., Kraberger, S., Stainton, D., Breitbart, M., and Varsani, A. (2012a). Diverse circular ssdna viruses discovered in dragonflies (odonata: Epiprocta). Journal of General Virology, 93:2668-2681.

Rosario, K., Duffy, S., and Breitbart, M. (2012b). A field guide to eukaryotic circular single-stranded dna viruses: insights gained from metagenomics. Archives of virology, 157(10):1851-1871.

Rosario, K., Nilsson, C., Lim, Y. W., Ruan, Y., and Breitbart, M. (2009). Metagenomic analysis of viruses in reclaimed water. Environmental Microbiology, 11(11):2806-20.

Roux, S., Enault, F., Bronner, G., Vaulot, D., Forterre, P., and Krupovic, M. (2013). Chimeric viruses blur 
the borders between the major groups of eukaryotic single-stranded dna viruses. Nature Communications, 4.

Roux, S., Krupovic, M., Poulet, A., Debroas, D., and Enault, F. (2012). Evolution and diversity of the microviridae viral family through a collection of 81 new complete genomes assembled from virome reads. PloS one, 7(7):e40418.

Sano, D., Kensuke, F., Yoshida, Y., and Omura, T. (2003). Detection of enteric viruses in municipal sewage sludge by a combination of the enzymatic virus elution method and rt-pcr. Water Research, 37(14):3490-3498.

Schmieder, R. and Edwards, R. (2012). Insights into antibiotic resistance through metagenomic approaches. Future Microbiology, 7(1):73-89.

Schmitz, J. E., Schuch, R., and Fischetti, V. A. (2010). Identifying active phage lysins through functional viral metagenomics. Applied and Environmental Microbiology, 76(21):7181-7.

Shapiro, O. H. and Kushmaro, A. (2011). Bacteriophage ecology in environmental biotechnology processes. Current Opinion in Biotechnology, 22:449-455.

Shapiro, O. H., Kushmaro, A., and Brenner, A. (2009). Bacteriophage predation regulates microbial abundance and diversity in a full-scale bioreactor treating industrial wastewater. The ISME journal, 4(3):327-336.

Śliwa-Dominiak, J., Suszyńska, E., Pawlikowska, M., and Deptuła, W. (2013). Chlamydia bacteriophages. Arichives of Microbiology, 195(10-11):765-771.

Svraka, S., Rosario, K., Duizer, E., van der Avoort, H., Breitbart, M., and Koopmans, M. (2010). Metagenomic sequencing for virus identification in a public-health setting. Journal of General Virology, 91(Pt 11):2846-56.

Tamaki, H., Zhang, R., Angly, F. E., Nakamura, S., Hong, P.-Y., Yasunaga, T., Kamagata, Y., and Lui, W.-T. (2012). Metagenomic analysis of dna viruses in a wastewater treatment plant in tropical climate. Environmental Microbiology, 14(2):441-452.

Thurber, R. V. (2009). Current insights into phage biodiversity and biogeography. Current opinion in microbiology, 12(5):582-587.

van den Brand, J., van Leeuwen, M., Schapendonk, C., Simon, J., Haagmans, B., Osterhaus, A., and Smits, S. (2012). Metagenomic analysis of the viral flora of pine marten and european badger feces. Journal of virology, 86(4):2360-2365.

Wichman, H., Badgett, M., Scott, L., Boulianne, C., and Bull, J. J. (1999). Different trajectories of parallel evolution during viral adaptation. Science. 